\title{
Microwave absorption studies in field-cooled hafnium doped YBaCuO
}

\author{
A G VEDESHWAR ${ }^{\dagger}$, Md SHAHABUDDIN, H D BIST, \\ S K AGARWAL* and A V NARLIKAR* \\ Department of Physics, Indian Institute of Technology, Kanpur 208016, India \\ *National Physical Laboratory, New Delhi 110012, India.
}

\begin{abstract}
The differential microwave absorption is studied in the field-cooled samples. Flux-trapping during field-cooling is clearly demonstrated. Glassy behaviour starts appearing for fields greater than the lower intergranular critical field revealed by the difference between field-cooled and zero-field-cooled cases. Results can be understood in terms of superconducting cluster model.
\end{abstract}

Keywords. Microwave absorption; Hf doping; field cooling; glassy behaviour; superconducting cluster.

\section{Introduction}

The new class of superconductors with higher $T_{c}$ posed many puzzles in understanding them in a generalized picture, in contrast to the conventional ones. However, the structure, chemical composition or oxygen deficiency etc in these materials play an important role on physical properties. In their early experiment of measuring susceptibility in LaBaCuO, Muller et al (1987a) measured the susceptibility in zero field-cooled (ZFC) and field-cooled (FC) samples in the spirit of spin glass experiments (Binder and Young 1986). They observed a notable difference between the two cases just like spin glasses and proposed the glassy nature of these materials. However, a giant flux creep was suggested in explaining the exponential decay of magnetization with time (Mota et al 1988) and their differences in ZFC and FC cases (Krusin-Elbaum et al 1988; Yeshurun and Malozemoff 1988). Flux creep picture is merely a phenomenological approach to the glassy behaviour for relatively short times and low temperatures (Morgenstern 1989). Therefore, we present here our results of microwave absorption (MA) measurements in field-cooled Hf-doped 123 sample showing the glassy behaviour.

\section{Experimental}

Samples were prepared by the well-known solid-state oxide mixing, sintering and annealing technique, i.e. through the ceramic route. These Hf-doped samples were studied systematically by resistivity measurements, X-ray, SEM, density measurements, and microwave absorption (Vedeshwar et al 1989). $T_{c}$ was not affected much up to $2 \%$ Hf doping. A Varian E109 X-band EPR spectrometer along with a set of Helmoltz coil with constant current source was used to record MA spectra. The sample was cooled in the desired field, the MA spectra recorded and was then exposed

\footnotetext{
'For correspondence: Materials Science Laboratory, Indira Gandhi Centre for Atomic Research, Kalpakkam 603102 . India
} 
to the negative field and again the spectra were recorded. The field was measured with an accuracy $0.05 \mathrm{mT}$ with precision digital Gaussmeter. The recording parameters of the spectrometer were suitably chosen to resolve the spectra. All spectra were recorded at $77 \mathrm{~K}$ using a specially homemade cryostat.

\section{Results and discussion}

Microwave absorption is interpreted as due to fluxon pinning and depinning in the critical state (Blazey et al 1988). Also, it is very sensitive to the microstructure-like grain size, impurity phases etc (Vedeshwar et al 1989). Therefore, we have chosen this probe to study the FC case at very low fields. The actual recorded MA spectra in $\mathrm{ZFC}$ and FC samples are shown in figure 1. The continuous curve shows the first recording and the dotted one shows the second recording. In the ZFC case, it can be noted that in the second run (dotted) signal height decreases because of trapped flux. In the FC case, each spectrum is labelled at which the field was cooled. In the second run, after exposing the sample to $-4 \mathrm{mT}$, the signal recovers towards original ZFC spectrum indicating depinning of trapped flux. We have shown the parameters of these spectra like peak position $\left(H_{m}\right)$, signal height $\left(h_{p p}\right)$ and area under the hysteresis

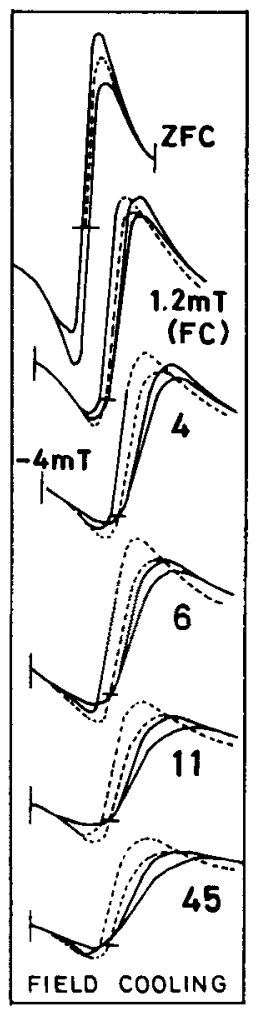

Figure 1. Actual recorded differential microwave absorption spectra in ZFC and FC samples at $77 \mathrm{~K}$. Numbers at left represent the field at which the sample was cooled. Recording conditions: modulation amplitude $=0.1 \mathrm{mT}$ at $100 \mathrm{kHz}$, gain $=10^{2}$ and microwave power $=0.2 \mathrm{~mW}$ at $9.2 \mathrm{GHz}$. 


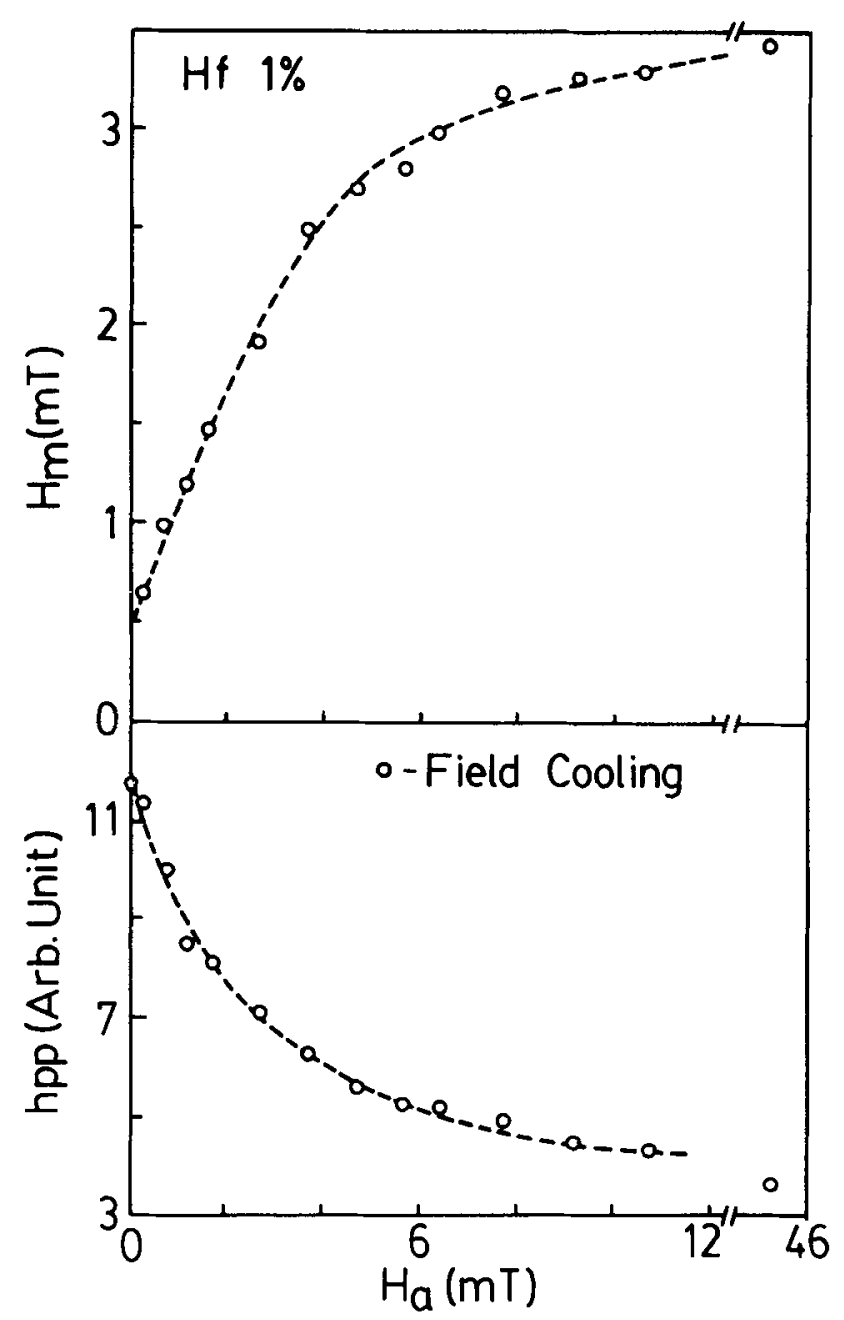

Figure 2. Parameters derived from figure 1 as a function of cooling field.

loop $(\Delta A)$ as a function of cooling field $\left(H_{a}\right)$ in figures 2 and 3 . It can be seen that $H_{m}$ shifts to higher fields and saturates around $6 \mathrm{mT} . H_{m}$ is identified as $H_{c 1}^{*}$ and can be used to determine the superconducting loop area projected normal to the applied field according to the relation $S=\phi_{0} / 2 H_{c 1}^{*}$ (Blazey et al 1987). It can be noted that the loop area decreases as a function of the cooling field. The signal height decreases with increasing cooling field showing reduction in loss which is due to the pinning of vortices. Figure 3 depicts the decrease in hysteresis loop area because of the trapping of fluxes during FC process. All these parameters saturate around $6 \mathrm{mT}$. This field can be identified as the upper intergranular critical field beyond which grains are decoupled and intragranular properties start to appear. The difference between ZFC and FC starts for fields greater than $H_{m}$, that is the lower intergranular critical field in conformity with the observation of Muller et al (1987b) and with theoretical predictions of Aksenov and Sergeenkov (1989). It is pointed out that glassy 


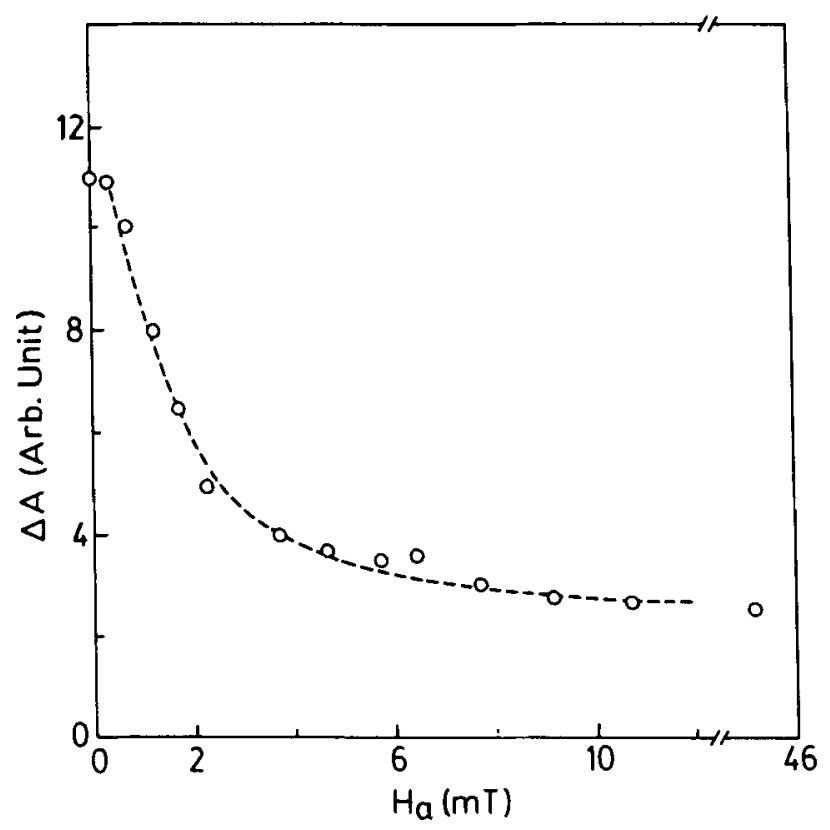

Figure 3. Area under the hysteresis loops (from figure 1) as a function of cooling field.

effects are predicted for temperatures between $70 \%$ and $95 \% T_{c}$ and the magnetic fields in the range of $0.03 \mathrm{~T}$ to $0.2 \mathrm{~T}$ (Morgenstern 1989). However, our observation is consistent with the above specified temperature range. We have observed the glassy nature at much lower fields $\left(\sim 0.5 \mathrm{mT} \geqslant H_{m}\right)$. Also, if we expose the sample to the negative field equal to the cooling field, all fluxes are not depinned revealed by the slight difference between the ZFC and FC processes suggesting some intrinsic pinning. This may be due to the inhomogeneous coupling strengths between different grains arising from the inhomogeneous distribution of grain sizes.

\section{Conclusions}

Using differential microwave absorption we have shown flux-trapping during FC procedure. During the FC process superconducting cluster chooses stable configuration to form the loop satisfying flux quantization. Glassy behaviour appears for fields greater or equal to $H_{m}$. A detailed analysis is being carried out and will be published elsewhere.

\section{Acknowledgements}

The authors gratefully acknowledge financial support from the Department of Science and Technology, New Delhi. We thank G S Thapa and Dinesh Kanojia for technical help. 


\section{References}

Akesnov V L and Sergeenkov S A 1989 Physica C156 18,235

Binder K and Young A P 1986 Rev. Mod. Phys. 58 801

Blazey K W, Muller K A, Bednorz J G, Berlinger W, Amoretti G, Buluggiu E, Vera A and Matacotta F C 1987 Phys. Rev. B36 7241

Blazey K W, Portis A M and Bednorz J G 1988 Solid State Commun. 651153 and references therein

Krusin-Elbaum L, Malozemoff A P, Yeshurun Y, Cronemeyer D C and Holtzberg F 1988 Physica C153 1469 and references therein

Morgenstern I 1989 IBM J. Res. Dev. 33307

Mota A C, Pollini A, Visani P, Muller K A and Bednorz J G 1988 Physica C153 67

Muller K A, Takashige M and Bednorz J G 1987a Phys. Rev. Lett. 581143

Muller K A, Blazey K W, Bednorz J G and Takashige M 1987b Physica B148 149

Vedeshwar A G, Shahbuddin Md, Chand P, Bist H D, Agarwal S K, Moorthy V N, Rao C V N and Narlikar A V 1989 Physica C158 385

Yeshurun Y and Malozemoff A P 1988 Phys. Rev. Lett. 602202 